\title{
Position Independent Wearable 6.78 MHz Near-Field Radiative Wireless Power Transfer using Electrically-Small Embroidered Textile Coils
}

\author{
M. Wagih ${ }^{1}$, A. O. Komolafe ${ }^{1}$, B. Zaghari ${ }^{1}$ \\ ${ }^{1}$ School of Electronics and Computer Science, University of Southampton, United Kingdom
}

\begin{abstract}
Coupled wireless power transfer (WPT) has been widely used for near-field high-efficiency WPT applications. However, the efficiency of the WPT link is highly sensitive to separation and alignment, and is prone to over-coupling, making it unsuitable for mobile systems with unknown or loose coupling such as wearables. While ultra-high frequency (UHF) and microwave radiative WPT $(0.3-3 \mathrm{GHz})$ enables meters-long separation between the transmitter and the receiver, free space propagation losses, and rectification inefficiencies, adversely limit the end-to-end efficiency of the WPT link. This work proposes radiative WPT, in the $6.78 \mathrm{MHz}$ license-free band, based on resonant electrically small antennas fabricated using embroidered textile coils, tuned using L-matching networks. The proposed WPT system achieves a stable forward transmission of $S_{21}>-17 \mathrm{~dB}$ and $\mathrm{S}_{21}>-28 \mathrm{~dB}$, independent of coil, separation on the XZ and XY planes respectively, in a $27 \mathrm{~m}^{3}$ volume space. The presented approach demonstrates the highest WPT-link efficiency, and promises higher end-to-end efficiency, compared to UHF WPT.
\end{abstract}

\section{Introduction}

Wireless Power Transfer (WPT) is an increasingly popular method of delivering power to an electronic system [1]. With established applications in the consumer electronics market and electric vehicle charging, the next generations of WPT will be based on resonant coupling to allow for increased separation between the transmitter and the receiver, in addition to non-coupled methods, through far-field propagation of WPT-specific waveforms. A particularly interesting platform for WPT is wearable e-textile applications [2], where establishing a real connection to the power supply is not feasible, and traditional energy harvesters require complex fabrication techniques to be integrated in textiles and flexible materials. Therefore, delivering power wirelessly to a wearable device represents a simple way of charging wearable nodes.

Strongly coupled magnetic resonance is commonly used for short range WPT in consumer electronics, while it offers improved ranges over inductive coupling, the coupling factor required between the coils to maintain high WPT efficiency implies significant constraints over the separation distances, size of coils and angular alignment. On the other hand, far-field WPT at UHF, microwave and millimetre-wave frequencies offers improved energy coverage and range, sacrificing on the wireless link's efficiency, and the RF-DC efficiency [3].

A hybrid approach combining sub-100 MHz WPT and radiative WPT could be adopted to mitigate the propagation losses and simplify the matching network design improving the maximum achievable rectification efficiency. This work proposes radiative WPT using impedance-matched resonant electrically small coils, at 6.78 MHz. The proposed frequency band and antenna design reduce the complexity and cost of designing a matching network and maintain a stable WPT efficiency regardless of the coil's separation. This paper discusses the motivation behind the radiative WPT at $6.78 \mathrm{MHz}$, the fabrication of fully-textile coils for WPT, and evaluates the performance of the system under different operation conditions.

\section{Radiative 6.78 MHz WPT and System Architecture}

The main challenges associated with UHF WPT are the propagation losses, proportional to the distance and frequency squared in as in (1), and the rectification inefficiency.

$$
\text { Friis Path Loss }(d B)=20 \log (d)+20 \log (f)+20 \log \left(\frac{4 \pi}{c}\right)-G_{t}-G_{r}
$$

To illustrate the WPT challenges at microwave frequencies, the propagation losses in the UHF bands have been calculated using (1). Furthermore, a low-barrier Schottky diode (SMS7630) has been simulated using harmonic balance in Keysight Advance Design Systems (ADS) to calculate its maximum achievable RF-DC efficiency assuming ideal impedance matching, at $0 \mathrm{dBm}$. Fig. 1 shows the decrease of the maximum RF-DC efficiency at microwave bands along with the logarithmic increase in propagation losses with frequency. 


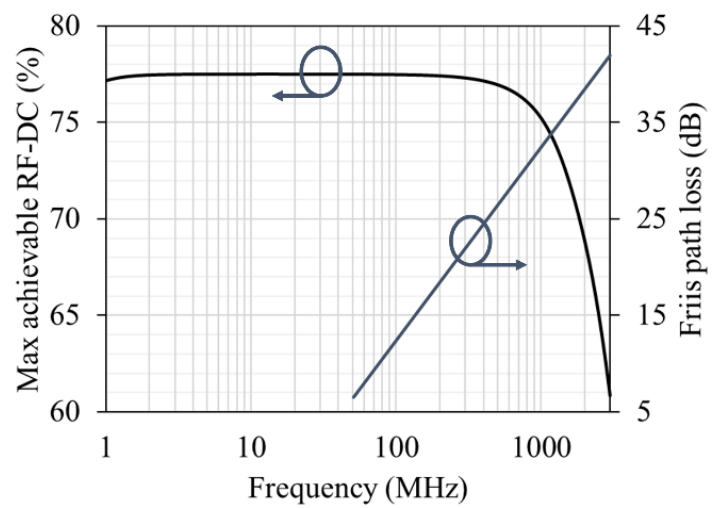

Fig. 1. Maximum achievable RF-DC efficiency simulated using non-linear harmonic balance, and propagation losses (Friis model at $1 \mathrm{~m}$ ) over frequency.

Motivated by overcoming the losses presented in Fig. 1, selecting a sub-10 MHz WPT carrier frequency becomes an apparent choice. The $6.78 \mathrm{MHz}$ Industrial Scientific and Medical (ISM) band is selected as the design frequency in this work. At $6.78 \mathrm{MHz}$ the specified limits by the Federal Communications Commission (FCC) are an electric field of $30 \mu \mathrm{V} / \mathrm{m}(-45.7 \mathrm{dBm})$, measured at 30 metres from the source. Moreover, in certain biomedical applications, the FCC has approved a waiver of the limit for up to $200.2 \mu \mathrm{V} / \mathrm{m}(-29.2 \mathrm{dBm})$ for a wirelessly-powered device demonstrating minimal interference with the surroundings [4]. While such low power limits constrain this WPT technology to ultra-low power applications, multiple systems have been presented with sub- $\mu \mathrm{W}$ power consumption for implants and biomedical applications [5].

The proposed system, shown in Fig. 2, utilizes electrically small coils to radiate power at $6.78 \mathrm{MHz}$, benefiting from lower propagation losses and free-positioning compared to UHF bands and coupled WPT, respectively. As the focus of this work is investigating the efficiency of the WPT link, the whole system is evaluated as a black box using its scattering-parameters (s-parameters) matrix, the forward transmission $\left(\mathrm{S}_{21}\right)$ is the main parameter measured in this work which can be used to calculate the WPT efficiency using $\eta_{\mathrm{WPT}}=$ $\left|S_{21}\right|^{2}$. Utilising s-parameters for evaluating WPT through an electromagnetic black box or for near field applications has been previously widely used as in [6].

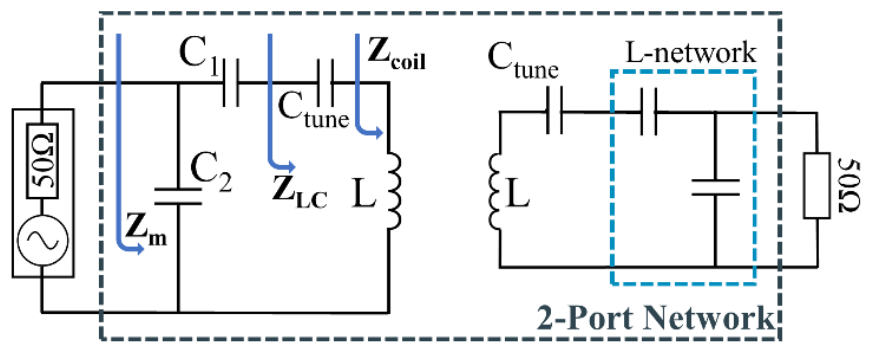

Fig. 2. The proposed system architecture showing the impedance matching of the coils.

\section{Textile WPT Coils Design and Fabrication}

The main area-consuming component in a WPT system is the coil or the antenna, where the size can affect the maximum achievable WPT efficiency. Therefore, the coils are the only component from Fig. 2 implemented on textile, as the capacitors could be realised using low footprint surface mount components or on-chip capacitor integrated with the electronics on the same chip. In order to fabricate the coil shown in Fig. 3, silkcoated Litz wires are embroidered to a textile substrate using an automated embroidery machine using a CAD model designed using commercial PCB design package. While the geometry of the coils is not critical in this work, due to the external impedance matching based on the measured impedance, the coil dimensions have been chosen to increase the inductance reducing the size of $\mathrm{C}_{\text {tune }}$ required to achieve resonance. However, the performance of the WPT is not expected to be affected significantly by changes in the coil geometry. To illustrate, the coil will always operate as an electrically small antenna and the minor changes in the geometry remain insignificant compared to the wavelength at $6.78 \mathrm{MHz}$, which implies minimal effect on the antenna's radiation efficiency. 


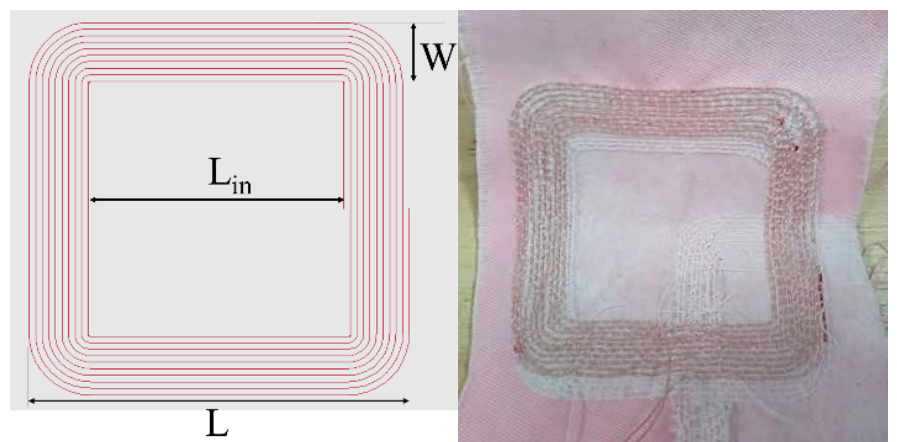

Fig. 3. Layout of the 10-turn coil (left) and photograph of the fabricated sample (right). Dimensions (mm): $W=10, L=60, L_{i n}=40$.

Fig. 4 shows the cross-section and a close-up view of the coils showing the Litz conductors; reducing the skin effect at higher frequencies.

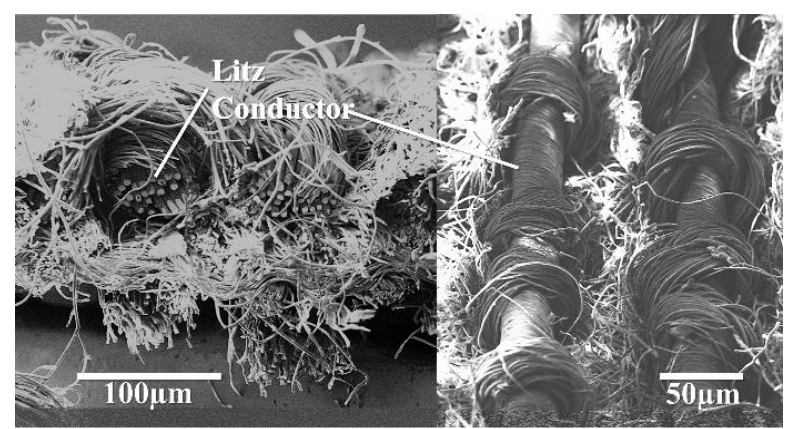

Fig. 4. Scanning electron microscope (SEM) cross-section (left) and surface (right) of the fabricated coil showing the conductive Litz wires.

\section{Electrically Small 6.78 MHz WPT Antennas}

For the coils to act as radiative antennas, the coils need to resonate at $6.78 \mathrm{MHz}$, and have their impedance matched to the source and load. The coils are tuned to resonate at $6.78 \mathrm{MHz}$ using a tuning capacitor calculated using equation 2. The input impedance of the tuned coil is then measured using a Vector Network Analyzer (VNA) to design a matching network, to allow the coils to radiate at $6.78 \mathrm{MHz}$.

$$
F_{\text {resonance }}=\frac{1}{2 \pi \sqrt{L C}}
$$

A capacitive L-matching network is designed to match $Z_{\mathrm{LC}}$ to $50 \Omega$, for compatibility with standard lab equipment, under real operation conditions, the matching network could be designed to match the coil to the impedance of the rectifier and the transmitter. Fig. 5 shows the impedance matching procedure on the smith chart, as well as the measured impedance of the matched coil, acting as an electrically small antenna.
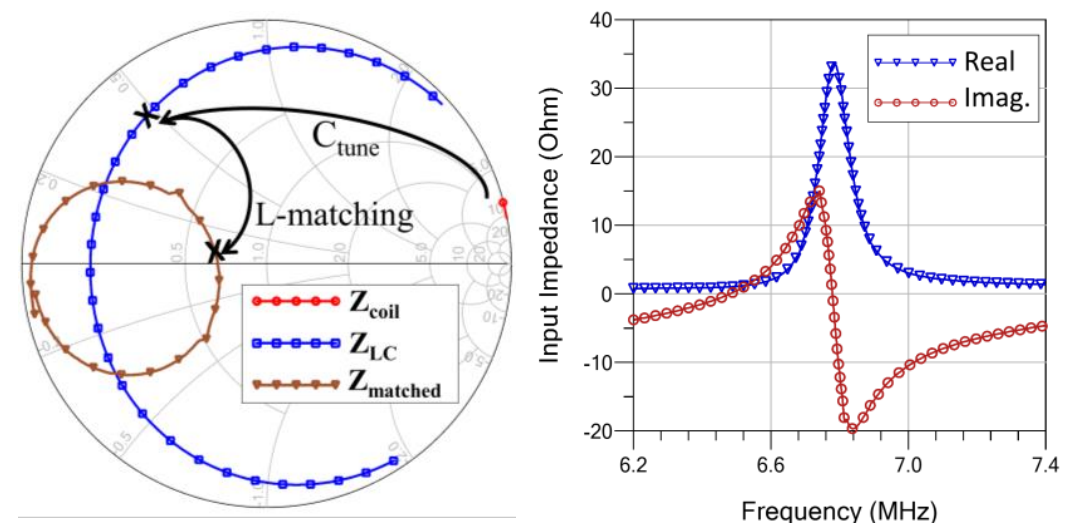

Fig. 5. Smith chart showing the measured input impedance of the coil (1-8 MHz) at different matching stages (left), and the input impedance of the matched coil (right).

The S(2,1) of the two coils and the L-matching network (the two-port network shown in Fig. 2) has been measured to evaluate the efficiency of the WPT link. A VNA is used measure the s-parameters of the network 
at variable coil separations. Fig. 6 , shows the coil radiating at $6.78 \mathrm{MHz}$, showing a $48 \mathrm{KHz} \mathrm{S}_{11}$ bandwidth, at $10 \mathrm{~cm}$ separation between the coils, demonstrating uncoupled WPT between the coils.

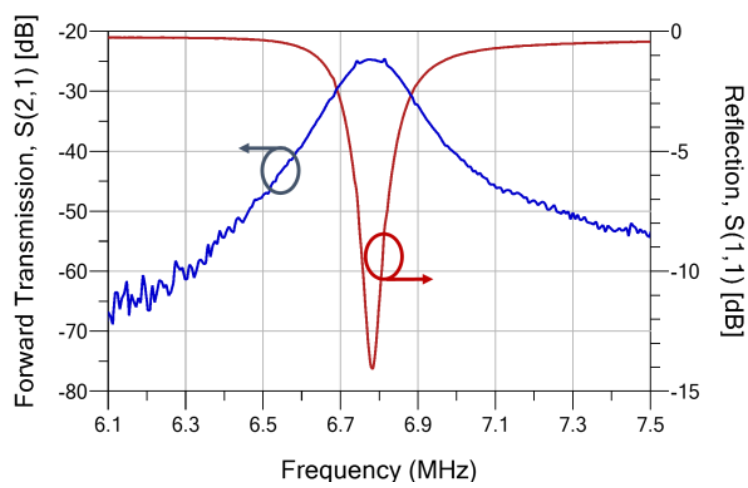

Fig. 6. Measured 2-port s-parameters of the matched coils at $10 \mathrm{~cm}$ separation

The separation between the coils has been studied under two conditions: fully aligned coils (vertical XZ plane) and full misalignment ( $180^{\circ}$ misalignment, $\mathrm{XY}$ plane) demonstrating the best and worst-case scenarios respectively; the measurements have been performed on multiple $3 \times 3 \mathrm{~m}^{2}$ planes in a $27 \mathrm{~m}^{3}$ volume to demonstrate the position-independence. In fig. 7, the system's $S_{21}$ is above $-17 \mathrm{~dB}$ and $-28 \mathrm{~dB}$ when aligned and misaligned respectively.

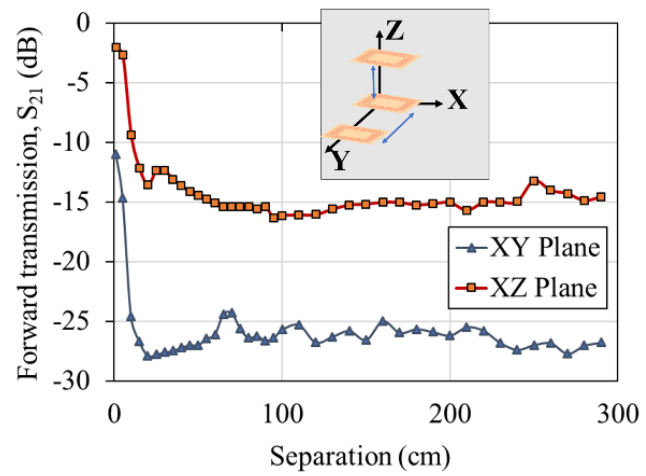

Fig. 7. Measured forward transmission in space of the proposed coils over variable coil separation on the XY and $\mathrm{XZ}$ planes.

While such behaviour is predicted for any isotropically radiating system, the position and separation independence achieved is unmatched by any coupled or uncoupled WPT system. Fig. 8 shows that at 6.78 MHz the WPT efficiency is the highest compared to UHF bands up to $3 \mathrm{GHz}$ at the same distance.

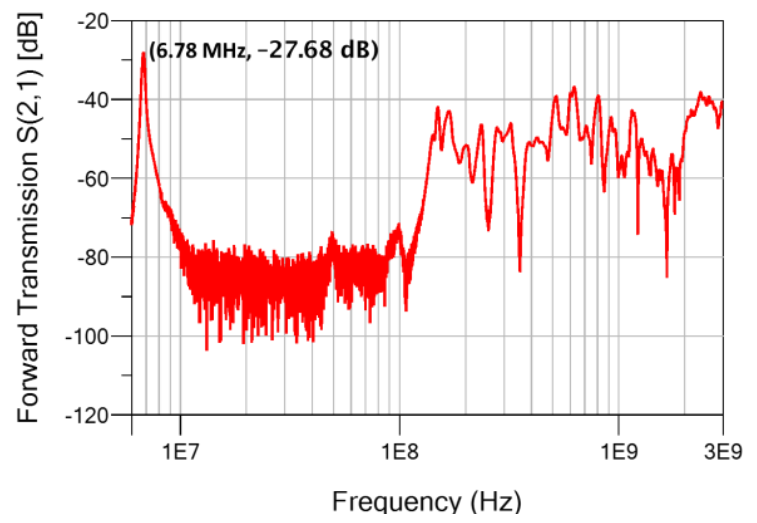

Fig. 8. Measured $\mathrm{S}_{21}$ from $6 \mathrm{MHz}$ to $3 \mathrm{GHz}$ showing the highest WPT efficiency at $6.78 \mathrm{MHz}$ compared to UHF bands.

\section{Conclusion}

This work has presented a new approach to separation and position independent WPT using a hybrid technique utilising radiative power transfer using electrically small antennas (coils). Radiative power transfer at $6.78 \mathrm{MHz}$ is presented for the first time demonstrating improved position and separation independence over 
near-field resonant WPT and microwave WPT. The validity of the approach has been demonstrated and the efficiency of the link experimentally characterized showing a constant forward transmission regardless of spatial separation.

Future work includes an investigation into the electric-field distributions around the coils, for transmitting effective amount of power to a load while complying with the FCC regulations. In addition, complete system design including a real power transmitter, and an impedance matched rectifier with a resistive load is to be presented.

\section{References}

[1] J. Garnica, R. A. Chinga, J. Lin, "Wireless Power Transmission: From Far Field to Near Field" Proceedings of the IEEE, vol. 101, no. 6 (2013) $1321-1331$.

[2] A. Komolafe et. al. "A smart cycling platform for textile based sensing and wireless power transfer in smart cities", Proceedings, E-textiles, (2019) London, U.K. (In press).

[3] B. Clerckx and E. Bayguzina "Waveform Design for Wireless Power Transfer" IEEE Trans. on Signal Processing, vol. 64, non 23, (2016), $6313-6328$.

[4] Federal Communication Commission "Federal Communications Commission Washington, D.C. 20554" online: https://apps.fcc.gov/edocs public/attachmatch/DA-09-2425A1.pdf accessed: September 2019.

[5] X. Zou, X. Xu, L. Yao, Y. Lian "A 1-V 450-nW Fully Integrated Programmable Biomedical Sensor Interface Chip" IEEE J. Solid State Circuits, vol. 44, no. 4 (2009), 1067 - 1077.

[6] H. Sun, W. Geyi "Optimum Design of Wireless Power Transmission Systems in Unknown Electromagnetic Environments" IEEE Access, vol. 5 (2017), 20198 - 20206.

Acknowledgement: This work was supported by the UK Engineering and Physical Sciences Research Council (EPSRC) under Grant EP/P010164/1.

Corresponding author: Mahmoud Wagih, School of ECS, University of Southampton, SO17 1BJ, U.K., +442380593234,.mahm1g15@ecs.soton.ac.uk 